\title{
Psicooncología
}

ISSN: 1696-7240

\section{Revisión sistemática sobre la eficacia de la Terapia de Aceptación y Compromiso (ACT) en el tratamiento psicológico de pacientes con cáncer}

\author{
Alba María García Rasero ${ }^{*}$, José Antonio Tamayo Hernández²
}

Recibido el 4 de junio de 2018 / Aceptado el 22 de diciembre de 2018

Resumen: Introducción: Aunque se han publicado muchos estudios que han intentado demostrar la eficacia de diferentes tratamientos psicológicos en pacientes con cáncer; hasta la fecha no se ha revisado la evidencia disponible sobre la eficacia de la Terapia de Aceptación y Compromiso (ACT) en estos pacientes. Objetivo: se pretende analizar sistemáticamente la evidencia empírica sobre la ACT en el tratamiento psicológico de los enfermos con cáncer. Método: se ha realizado una búsqueda en las bases de datos PubMed, Science Direct, Medline, Psychology and Behavioral Sciences Collection, Journal of Contextual Psychology y Cochrane Biblioteca. Resultados: De los 108 artículos encontrados, se han seleccionado para la revisión un total de 8 , que seguían un diseño experimental o cuasiexperimental en pacientes adultos diagnosticados de cáncer tratados con ACT. Conclusiones: Los resultados, aunque heterogéneos, sugieren mejorías a medio - largo plazo en las variables de ansiedad, depresión, aceptación, calidad de vida, distrés y flexibilidad psicológica. Además, la ACT ha resultado ser más eficaz que otras terapias psicológicas. De este modo, a pesar de las limitaciones encontradas en este estudio, se han obtenido resultados prometedores para futuras revisiones y/ o metaanálisis.

Palabras Claves: cáncer, Terapia de Aceptación y Compromiso, eficacia, familia, evidencia empírica.

\section{[en] Systematic review on the effectiveness of Acceptance and Commitment Therapy (ACT) in the psychological treatment of cancer patients}

\begin{abstract}
Introduction: Although many studies have been published that have tried to demonstrate the efficacy of different psychological treatments in patients with cancer; to date, the available evidence on the effectiveness of Acceptance and Commitment Therapy (ACT) in these patients has not been reviewed. Objetive: The aim is to systematically analyze the empirical evidence on ACT in the psychological treatment of patients with cancer. Method: A search was made in PubMed, Science Direct, Medline, Psychology and Behavioral Sciences Collection, Journal of Contextual Psychology, and Cochrane Library databases. Results: Among the 108 articles found, a total of 8 were selected for the review, which followed an experimental or quasi-experimental design in adult patients diagnosed with cancer treated with ACT. Conclusions: The results, although heterogeneous, suggest improvements in the medium - long term after ACT intervention in the symptoms of anxiety, depression, acceptance,

1 Alba María García Rasero. Centro Activa Psicología y Formación. Madrid. España

E-mail: alba.garciarasero@outlook.com

2 José Antonio Tamayo Hernández. Centro Universitario de Ciencias de la Salud San Rafael-Nebrija. Madrid. España.

E-mail: jtamayo@nebrija.es

* Dirección de correspondencia: Alba María García Rasero. Glorieta de Bilbao, 1, 1º Centro. 28004-Madrid. España. E-mail: alba.garciarasero@outlook.com
\end{abstract}


quality of life, distress and psychological flexibility. In addition, ACT has been found to be more effective compared to other psychological therapies. Thus, despite the limitations found in this study, promising results have been obtained for future reviews and / or meta-analyzes.

Keywords: cancer, acceptance and commitment therapy, efficacy, clinical evidence, family.

Sumario. 1. Introducción 2. Metodología 2.1. Análisis de datos 3. Resultados 3.1. Resultados del grupo experimental 3.2. Resultados comparativos entre el grupo experimental y el grupo control 4. Discusión 4.1. Limitaciones 5. Conclusiones 6. Referencias bibliográficas.

Cómo citar: García Rasero AM, Tamayo Hernández JA. Revisión sistemática sobre la eficacia de la Terapia de Aceptación y Compromiso (ACT) en el tratamiento psicológico de pacientes con cáncer. Psicooncología 2019; 16(1): 101-125. doi: 10.5209/PSIC.63651.

\section{Introducción}

La mayoría de las aproximaciones terapéuticas al tratamiento psicológico de pacientes con cáncer se han basado en intervenciones psicosociales o cognitivo-conductuales. En ellas, en especial desde estas últimas, se establece como objetivo del tratamiento el cambio o control de los pensamientos y emociones aversivos, cuya función se conceptualiza como causal respecto al comportamiento problemático que presenta el paciente ${ }^{(1)}$. Por tanto, esta perspectiva asumiría como premisa la eliminación de los eventos privados negativos como requisito indispensable de la salud psicológica $\mathrm{y}$, por ende, del tratamiento ${ }^{(2)}$.

Las terapias cognitivo-conductuales han demostrado ser particularmente eficaces en aquellos pacientes que utilizan un estilo de afrontamiento pasivo/evitativo o de negación $^{(3)}$ Sin embargo, también presentan algunas limitaciones, como no haber proporcionado hasta la fecha ningún dato o evidencia del mecanismo de acción por el que resultan eficaces. Es decir, aun demostrando ser eficaces, todavía no se conoce si su eficacia obedece a los componentes cognitivos, conductuales o a una conjunción de $\operatorname{ambos}^{(4)}$. Además, se ha podido comprobar que, tras finalizar la intervención, la mejoría en la sintomatología psicológica de los pacientes con cáncer se atenúa con el paso del tiempo ${ }^{(5)}$

En contraste, en los últimos años ha surgido un elenco de nuevas terapias psicológicas, popularizadas como "contextuales" o de "tercera generación"(6), que siguen una perspectiva funcional y contextual para explicar de forma empírica la relación de la cognición y el lenguaje con los patrones de comportamiento observables $^{(7)}$. De todas ellas, quizás pueda considerarse la Terapia de Aceptación y Compromiso (ACT) como paradigmática ${ }^{(1)}$, al ser la más completa, mejor desarrollada y que cuenta con un fundamento teórico más sólido, sustanciado en la Teoría del Marco Relacional (RFT), como explicación del lenguaje y de la cognición humana desde los principios del análisis funcional de la conducta ${ }^{(7-9)}$.

Para la ACT, la naturaleza verbal del ser humano impide la posibilidad de evitar completamente la irrupción de cogniciones (ya sean acerca del pasado, presente o futuro, tales como los recuerdos, valoraciones o anticipaciones), así como de que aparezcan cargadas de emociones de distinta valencia afectiva. Por consiguiente, dado que la agenda establecida culturalmente apunta a la necesidad de "sentirse bien" como criterio de normalidad psicológica, la persona se funde en un sinfín de acciones 
que intentan acabar con los pensamientos y emociones etiquetados socialmente como negativos ${ }^{(8)}$. Este proceso, denominado evitación experiencial o inflexibilidad psicológica, consistiría en un patrón de conducta generalizado cuya función es evitar el contacto con una experiencia privada aversiva concreta e intentar controlar su forma o la frecuencia de aparición. Aunque este comportamiento genera un alivio inmediato, a largo plazo resulta contraproducente, improductivo y limitante en la actuación de la persona en la consecución de lo que es importante en su vida ${ }^{(10,6,5)}$.

Por lo tanto, la ACT estaría indicada para el tratamiento de todos aquellos problemas psicológicos caracterizados por un patrón de evitación experiencial cronificado y limitante ${ }^{(5)}$, como puedan ser los trastornos afectivos, trastornos de ansiedad, trastornos de la conducta alimentaria, trastornos por consumo de sustancias, trastornos psicóticos, dolor crónico, o trastornos adaptativos secundarios a una enfermedad médica, como el cáncer ${ }^{(11)}$.

En el caso del cáncer, desde el momento en que un paciente recibe el diagnóstico, pasa por innumerables situaciones nuevas, llenas de incertidumbre, que suponen una alta carga estresante por las preocupaciones, temores y el dolor asociados ${ }^{(2)}$; al igual que la presencia de síntomas depresivos ${ }^{(12)}$. En este sentido, cabe destacar también, tal y como se recoge en el metaanálisis de Mitchell et al. ${ }^{(13)}$, que el 12,9-29,8\% de los pacientes con cáncer sufren depresión, y entre el 5,1-17\% experimentan ansiedad. Tanto la ansiedad como la depresión pueden influir de manera independiente en la salud de los pacientes oncológicos, constituyendo, por tanto, un reto actual la gestión eficaz de estos síntomas ${ }^{(14)}$. A esto hay que añadir que en no pocas ocasiones los pacientes pierden el contacto con amigos y familiares por miedo a la reacción que estos puedan tener ${ }^{(15)}$.

Por otra parte, el proceso de tratamiento médico del cáncer es complejo y habitualmente se asocia con complicaciones que influyen a nivel físico, psicológico y social, tanto en los pacientes como en sus familiares ${ }^{(16)}$, que tienen que proporcionar cuidado y apoyo emocional al enfermo, obligándoles a asumir nuevas responsabilidades ${ }^{(17)}$. Para los hijos de padres diagnosticados de cáncer, la enfermedad se convierte en un estresor de primera magnitud y de características únicas, que llega a comprometer seriamente su bienestar emocional. Así, por ejemplo, pueden adolecer de no tener cubiertas muchas de sus necesidades, como el soporte emocional familiar, puesto que toda la atención del entorno se centra en el enfermo; o también pueden sufrir la falta de comprensión de la enfermedad que sufre alguno de sus progenitores, entre otros problemas ${ }^{(18)}$. Tal y como mencionan en su estudio de revisión Visser et al. ${ }^{(19)}$, los niños en edad escolar, cuyo progenitor está enfermo de cáncer, presentan más miedo a la enfermedad y al tratamiento. En el caso de los adolescentes, pueden mostrar tanto problemas externalizantes (i.e. problemas académicos, dificultades para dormir, dolores de estómago) como internalizantes (i.e. síntomas depresivos y ansiosos) ${ }^{(18)}$.

Se ha observado, además, que el diagnóstico de cáncer en un miembro de la pareja también provoca un desajuste psicológico en el otro miembro ${ }^{(20)}$. De hecho, en muchas ocasiones los roles de la pareja cambian para poder cubrir todas las necesidades de los pacientes con cáncer, sacrificando sus actividades habituales y sus aficiones ${ }^{(21)}$.

En cuanto a la intervención, han sido muchos los estudios realizados con el fin de demostrar la eficacia de los diferentes tratamientos psicológicos en el cáncer. Así, por ejemplo, la terapia cognitivo-conductual ha evidenciado su efectividad 
en los síntomas psicopatológicos que suelen presentar los pacientes ${ }^{(12)}$, incluyendo para ello, en la mayoría de sus programas, técnicas para el control del estrés y la resolución de conflictos ${ }^{(14)}$.

Sin embargo, todas estas estrategias vuelven a hacer hincapié en el control y reducción de los síntomas, tales como temores, penas y padecimientos, para así sentirse mejor y eliminar la amenaza asociada al cáncer ${ }^{(5)}$. Por el contrario, conseguir distanciarse de los propios pensamientos (defusión cognitiva), evitando el control emocional y cognitivo, ha resultado ser mucho más eficaz en el tratamiento de los síntomas psicológicos en los enfermos de cáncer que el protocolo $\mathrm{TAU}^{3}$, de orientación cognitivo-conductual, que incluye técnicas de relajación, resolución de problemas y reestructuración cognitiva ${ }^{(22)}$. En esta misma dirección, Páez et al. ${ }^{(23)}$ concluyen que la ACT es más efectiva que los tratamientos cognitivo-conductuales, especialmente a largo plazo, y en la implicación de los pacientes hacia sus acciones valiosas.

Pese a ello, existen comparativamente muy pocas las investigaciones realizadas hasta la fecha sobre la aplicación de la ACT en pacientes con cáncer ${ }^{(9)}$, como también dirigidas a estudiar el papel de la aceptación de las alteraciones emocionales en estos pacientes, con el fin de que les ayude a manejarlos y a actuar según sus valores ${ }^{(12)}$.

\section{Metodología}

Debido a que hasta la fecha no se ha publicado ninguna revisión sistemática ni estudio meta-analítico que evalúe la eficacia de la ACT en los problemas psicológicos de los pacientes con cáncer, con este trabajo se pretende analizar la evidencia existente en la literatura científica a través de una revisión sistemática. Para ello, y como objetivos específicos, se proponen:

1) Conocer la eficacia/efectividad de la ACT en la mejora psicológica de los pacientes oncológicos.

2) Comparar la eficacia diferencial de la ACT respecto a otras terapias (psicológicas, psicosociales o farmacológicas).

Se consultaron las bases de datos: Medline complete, ScienceDirect, PubMed, Psychology and Behavioral Sciences Collection, y Cochrane plus; así como la revista Journal of Contextual Behavioral Science, de la Association for Contextual Behavioral Science. Para efectuar la búsqueda, se emplearon los siguientes términos documentales: "Acceptance and Commitment Therapy"/“Terapia de Aceptación y Compromiso", "cancer", "oncology"/“oncología", "effectiveness"/“efectividad", "efficacy"/“eficacia", "clinical evidence"/“evidencia clínica"; combinado por medio de los operadores booleanos AND y OR. Además, se realizó una búsqueda inversa en los artículos seleccionados, con el fin de identificar otros artículos que pudieran coincidir con los criterios de selección. El intervalo temporal de publicación de artículos cubría hasta septiembre de 2017.

Durante el proceso de búsqueda se revisaron los títulos y los resúmenes de cada artículo obtenido para fundar su inclusión en la revisión. De este modo, fueron seleccionados todos los artículos de temática psicológica, publicados tanto en inglés

3 Protocolo desarrollado por los investigadores basado en entrenamiento de relajación, resolución de problemas y reestructuración cognitiva. 
como en español, sin limitación de año, y cuya metodología fuera cuantitativa. De estos artículos, se incluyeron en la muestra aquellos estudios empíricos (con diseño experimental o cuasiexperimetal) que se ajustaran a los siguientes criterios:

- Pacientes diagnosticados de cualquier tipo de cáncer o supervivientes de cáncer

- Edad igual o mayor a 18 años (adultos)

- Que hubieran recibido tratamiento psicológico con ACT

Han sido excluidas las revisiones sistemáticas, los estudios de metaanálisis, las tesis doctorales, los artículos de caso único, y los artículos que no estuvieran disponibles a texto completo. De igual modo, también fueron excluidos aquellos artículos que no incluían a pacientes con cáncer o que no estaban tratados con la ACT. No se aplicaron otros límites (ver Figura 1).

Figura 1. Diagrama de flujo del proceso de búsqueda

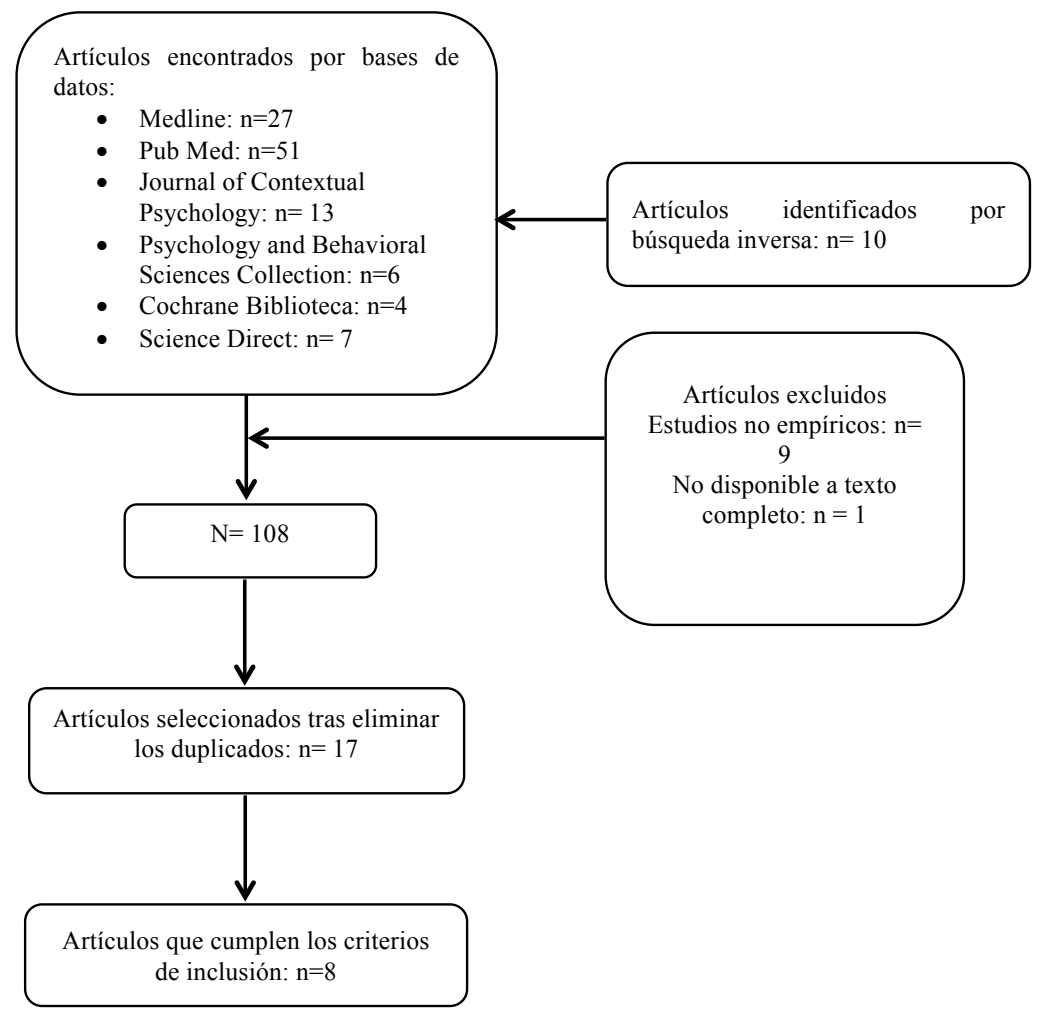

\subsection{Análisis de datos}

Se realizó un análisis descriptivo mediante la $d$ de Cohen de las medidas de ansiedad, depresión, distrés, aceptación, calidad de vida y expectativa de vida, incluidas en los artículos revisados como variables dependientes de la eficacia del tratamiento. 
A través de este estadístico se puede conocer el número de desviaciones típicas a las que se encuentran los resultados de los grupos experimentales y controles en el conjunto de los artículos, a fin de que las diferentes medidas de cada variable puedan ser comparables entre sí ${ }^{(24)}$. Para ello, se tuvieron en cuenta las medias y desviaciones típicas de las medidas de resultado de los diferentes artículos seleccionados. No obstante, antes de calcular dichas puntuaciones se han ajustado mediante el factor de corrección $[\mathrm{C}(\mathrm{gl})]^{4}$, para así conocer el valor real de las puntuaciones.

La interpretación de la magnitud de la $d$ se ha realizado de acuerdo con los valores de Cohen ${ }^{(25)}$, considerándose como efecto pequeño cuando los valores son menores de 0,20; medio hasta valores de 0,50; y grande en el caso de valores mayores de 0,80. El signo de la $d$ depende de la dirección en que se espera que cambie la variable con la intervención. Es decir, en aquellas variables en las que se espera que tras la intervención aumenten sus valores (i.e. calidad de vida), el signo será negativo si es efectiva; mientras que en aquellas donde se espera que se reduzcan (i.e. ansiedad), el signo será positivo.

\section{Resultados}

El proceso de búsqueda, siguiendo las ecuaciones, términos documentales y criterios de selección representados en la Tabla 1, arrojó finalmente un total de 8 artículos. De manera general destaca la heterogeneidad de todos los estudios recogidos en esta revisión, variando el número de participantes, el tipo de cáncer que padecen los pacientes, y las características de la intervención. Todos los estudios tienen en común el objetivo de mejorar en los participantes la angustia, el estado de ánimo (depresión, ansiedad y distrés), la calidad de vida, y la aceptación, entre otras variables.

Tabla1. Proceso de selección de artículos.

\begin{tabular}{|c|c|c|c|c|c|}
\hline Bases de Datos & $\begin{array}{c}\text { Ecuación } \\
\text { de } \\
\text { Búsqueda }\end{array}$ & $\begin{array}{c}\mathrm{N}^{\mathbf{0}} \text { de } \\
\text { referencias } \\
\text { encontradas }\end{array}$ & $\begin{array}{l}\mathrm{N}^{\circ} \text { de } \\
\text { referencias } \\
\text { tras leer } \\
\text { resumen } \\
\end{array}$ & $\begin{array}{c}\mathrm{N}^{0} \mathrm{de} \\
\text { referencias } \\
\text { tras lectura } \\
\text { crítica }\end{array}$ & $\begin{array}{c}\mathrm{N}^{\circ} \text { de } \\
\text { referencias } \\
\text { final }\end{array}$ \\
\hline MEDLINE & $\begin{array}{l}\text { "Acceptance and } \\
\text { Commitment } \\
\text { Therapy" AND } \\
\text { "Cancer" OR } \\
\text { "Oncology" AND } \\
\text { "Effectiveness" } \\
\text { OR "Clinical } \\
\text { Evidence" OR } \\
\text { "Efficacy" AND } \\
\text { "Family" OR "Rel- } \\
\text { atives" }\end{array}$ & 27 & 7 & 3 & 3 \\
\hline
\end{tabular}

4 El factor de corrección de continuidad es el ajuste de media unidad de medida para mejorar la exactitud cuando a una distribución discreta se le aplica una distribución continua ${ }^{(24)}$. 


\begin{tabular}{|c|c|c|c|c|c|}
\hline Bases de Datos & $\begin{array}{l}\text { Ecuación } \\
\text { de } \\
\text { Búsqueda }\end{array}$ & $\begin{array}{c}\mathrm{N}^{\mathrm{o}} \text { de } \\
\text { referencias } \\
\text { encontradas }\end{array}$ & $\begin{array}{l}\mathrm{N}^{\mathrm{o}} \mathrm{de} \\
\text { referencias } \\
\text { tras leer } \\
\text { resumen }\end{array}$ & $\begin{array}{c}\mathrm{N}^{0} \text { de } \\
\text { referencias } \\
\text { tras lectura } \\
\text { crítica }\end{array}$ & $\begin{array}{l}\mathrm{N}^{0} \mathrm{de} \\
\text { referencias } \\
\quad \text { final }\end{array}$ \\
\hline PUBMED & $\begin{array}{l}\text { “Acceptance and } \\
\text { Commitment } \\
\text { Therapy" AND } \\
\text { "Cancer" OR "On- } \\
\text { cology" OR "neo- } \\
\text { plasms" }\{\text { MeSH }\} \\
\text { AND "Effective- } \\
\text { ness" OR "Clinical } \\
\text { Evidence" OR "Ef- } \\
\text { ficacy" OR "out- } \\
\text { comes" }\{\text { MeSH }\} \\
\text { AND "Family" OR } \\
\text { "Relatives" }\end{array}$ & 51 & 2 & 2 & 2 \\
\hline $\begin{array}{l}\text { Journal of } \\
\text { Contextual } \\
\text { Psychology }\end{array}$ & "Cancer" & 13 & 7 & 3 & $\begin{array}{c}1+1 \\
\text { (búsqueda } \\
\text { inversa) }\end{array}$ \\
\hline $\begin{array}{l}\text { Psychology and } \\
\text { Behavioral } \\
\text { Sciences } \\
\text { Collection }\end{array}$ & $\begin{array}{l}\text { "Acceptance and } \\
\text { Commitment } \\
\text { Therapy" AND } \\
\text { "Cancer" OR } \\
\text { "Oncology" AND } \\
\text { "Effectiveness" OR } \\
\text { "Clinical Evidence" } \\
\text { OR "Efficacy" } \\
\text { AND "Family" OR } \\
\text { "Relatives" }\end{array}$ & 6 & 3 & 1 & 0 \\
\hline $\begin{array}{l}\text { Cochrane } \\
\text { Biblioteca }\end{array}$ & $\begin{array}{l}\text { "Acceptance and } \\
\text { Commitment } \\
\text { Therapy" AND } \\
\text { "Cancer" OR } \\
\text { "Oncology" AND } \\
\text { "Effectiveness" OR } \\
\text { "Clinical Evidence" } \\
\text { OR "Efficacy" }\end{array}$ & 4 & 0 & 0 & 0 \\
\hline \multirow[t]{2}{*}{$\begin{array}{l}\text { Science } \\
\text { Direct }\end{array}$} & $\begin{array}{l}\text { "Acceptance and } \\
\text { Commitment Ther- } \\
\text { apy" AND "Can- } \\
\text { cer" }\end{array}$ & 7 & 1 & 1 & 1 \\
\hline & & & & & Total $=$ \\
\hline
\end{tabular}

En primer lugar, y como puede observarse en la Tabla 2, el tipo de cáncer que aparece estudiado con más frecuencia es el cáncer de mama (75\%); seguido de otros tumores (genitourinario, cabeza, garganta, linfoma, ovarios pulmón y estómago: $12,5 \%)$ y de tumores no especificados $(12,5 \%)$.

Por otra parte, también se encuentran diferencias en cuanto a la utilización del grupo de control en los 8 estudios. En dos de ellos, el grupo control es sometido a otro tipo de terapia distinta a la ACT, con el que se comparan los resultados (terapia cognitivo- conductual); en cuatro estudios, el grupo control no recibe ningún tipo de 
terapia alternativa ( en sus investigaciones. La asignación de los participantes a cada grupo se hizo al azar en todos los estudios que cuentan con un grupo control (diseño experimental).

No se hallaron estudios que utilizaran como grupo experimental ni control a pacientes que recibieran otro tratamiento psicológico distinto al cognitivoconductual, como tampoco intervención psicosocial ni psicofarmacológica.

En cuanto a la evaluación, existen variaciones respecto al momento en que se realiza, aunque todos los artículos cuentan con una medida pre y post-tratamiento. Algunos de esos artículos incluyen, además, una medida de línea base antes de comenzar el tratamiento. De los 8 artículos seleccionados, en uno de ellos se toman medidas de seguimiento a los 3, 6 y 12 meses de finalizar el tratamiento. Otro estudio presenta dos medidas de seguimiento, al cabo de 1 mes y a los 3 meses. En dos artículos sólo se realiza una medida de seguimiento, a los 3 o a los 6 meses después de la primera medida. Por último, en dos de los estudios se tomaron solamente medidas durante la intervención, y otros dos no cuentan con ninguna medida de seguimiento.

Las principales variables dependientes que fueron extraídas de los artículos son: el nivel de angustia, el estado de ánimo (depresión, ansiedad y distrés), la calidad de vida (tanto de los pacientes de cáncer, como de los familiares con los que conviven), y la flexibilidad psicológica (entendida como la capacidad para experimentar sentimientos, emociones y pensamientos de manera plena, sin tratar de evitarlos $)^{(6)}$. De manera secundaria, en algunos de ellos también se tienen en cuenta las expectativas vitales del paciente, lo que guarda relación con el proceso de clarificación de valores para el discernimiento de las trayectorias valiosas, objetivos $\mathrm{y}$ acciones en esas trayectorias de la vida de pacientes con cáncer ${ }^{(7)}$; y el control emocional, es decir, los intentos del paciente de evitar sentimientos de ansiedad y preocupaciones propios de la enfermedad ${ }^{(6)}$.

Tabla 2. Descripción de los estudios del tratamiento de la ACT en pacientes con cáncer

\begin{tabular}{|c|c|c|c|c|c|c|}
\hline Autores & Patología & Participantes & $\begin{array}{l}\text { Grupo } \\
\text { control }\end{array}$ & $\begin{array}{l}\text { Evaluación y } \\
\text { Seguimiento }\end{array}$ & Resultados & Limitaciones \\
\hline $\begin{array}{l}\text { Páez et } \\
\text { al. }^{(23)}\end{array}$ & $\begin{array}{l}\text { Cáncer de } \\
\text { Mama }\end{array}$ & $\begin{array}{l}12 \text { mujeres } \\
\text { Edad: } 42-50 \\
\text { años } \\
\text { (Media=46,58 } \\
\text { años; DT }=2,87 \text { ) }\end{array}$ & $\mathrm{COG}^{5}$ & $\begin{array}{l}\text { Pre y post-tra- } \\
\text { tamiento, y } 3 \text {, } \\
6 \text { y } 12 \text { meses } \\
\text { después. }\end{array}$ & $\begin{array}{l}\text { Mayor eficacia } \\
\text { de la ACT en } \\
\text { las variables } \\
\text { ansiedad, de- } \\
\text { presión, cali- } \\
\text { dad de vida, y } \\
\text { en el cambio } \\
\text { de los com- } \\
\text { portamientos } \\
\text { en áreas valio- } \\
\text { sas afectadas a } \\
\text { largo plazo (1 } \\
\text { año). No hay } \\
\text { diferencias a } \\
\text { corto plazo. }\end{array}$ & $\begin{array}{l}\text { Se desconoce } \\
\text { qué proce- } \\
\text { dimiento en } \\
\text { particular fue } \\
\text { responsable } \\
\text { de la mayor } \\
\text { eficacia de la } \\
\text { ACT. } \\
\text { Muestra redu- } \\
\text { cida y sesgada, } \\
\text { extraída de un } \\
\text { entorno muy } \\
\text { concreto. }\end{array}$ \\
\hline
\end{tabular}




\begin{tabular}{|c|c|c|c|c|c|c|}
\hline Autores & Patología & Participantes & $\begin{array}{l}\text { Grupo } \\
\text { control }\end{array}$ & $\begin{array}{l}\text { Evaluación y } \\
\text { Seguimiento }\end{array}$ & Resultados & Limitaciones \\
\hline $\begin{array}{l}\text { Burke et } \\
\text { al. }{ }^{(27)}\end{array}$ & $\begin{array}{l}\text { Padres con } \\
\text { hijos diag- } \\
\text { nosticados } \\
\text { de cáncer o } \\
\text { que hayan } \\
\text { superado } \\
\text { una opera- } \\
\text { ción car- } \\
\text { diaca. }\end{array}$ & $\begin{array}{l}19 \text { padres, } 5 \\
\text { parejas com- } \\
\text { prometidas, } 7 \\
\text { madres casadas } \\
\text { (asistían solas a } \\
\text { las sesiones) y } 2 \\
\text { madres solteras. }\end{array}$ & $\begin{array}{l}\text { Sin } \\
\text { grupo } \\
\text { control }\end{array}$ & $\begin{array}{l}\text { Pre y post } \\
\text { tratamiento, y } \\
6 \text { meses des- } \\
\text { pués. }\end{array}$ & $\begin{array}{l}\text { Resultados } \\
\text { prometedores } \\
\text { en el estrés, } \\
\text { la flexibilidad } \\
\text { psicológica y } \\
\text { mindfulness }{ }^{6} \text { a } \\
\text { medio y largo } \\
\text { plazo tras la } \\
\text { intervención } \\
\text { en } \mathrm{TAB}^{7} \text {. }\end{array}$ & $\begin{array}{l}\text { Muestra muy } \\
\text { pequeña. No } \\
\text { hay grupo } \\
\text { control. }\end{array}$ \\
\hline $\begin{array}{l}\text { Ghasemi } \\
\text { et al. }{ }^{(28)}\end{array}$ & $\begin{array}{l}\text { Cáncer de } \\
\text { mama }\end{array}$ & $\begin{array}{l}24 \text { participantes } \\
\text { Edad: } 35-55 \\
\text { años. }\end{array}$ & $\begin{array}{l}\text { Sin in- } \\
\text { terven- } \\
\text { ción }\end{array}$ & $\begin{array}{l}\text { Pre y } \\
\text { post-tratam- } \\
\text { iento. }\end{array}$ & $\begin{array}{l}\text { ACT fue efec- } \\
\text { tiva a la hora } \\
\text { de crear espe- } \\
\text { ranza en los } \\
\text { pacientes. Re- } \\
\text { sultados posi- } \\
\text { tivos sobre la } \\
\text { salud mental, } \\
\text { la ansiedad, } \\
\text { los síntomas } \\
\text { depresivos y } \\
\text { la manera de } \\
\text { relacionarse } \\
\text { con los pensa- } \\
\text { mientos. }\end{array}$ & $\begin{array}{l}\text { No se especi- } \\
\text { fican. }\end{array}$ \\
\hline $\begin{array}{l}\text { Mohab- } \\
\text { bat-Bahar } \\
\text { et al. }^{(16)}\end{array}$ & $\begin{array}{l}\text { Cáncer de } \\
\text { mama }\end{array}$ & $\begin{array}{l}30 \text { pacientes } \\
\text { Edad: } 30-65 \\
\text { años }\end{array}$ & $\begin{array}{l}\text { Sin in- } \\
\text { terven- } \\
\text { ción }\end{array}$ & $\begin{array}{l}\text { Pre y } \\
\text { post-tratam- } \\
\text { iento }\end{array}$ & $\begin{array}{l}\text { La ACT obtie- } \\
\text { ne resultados } \\
\text { positivos en la } \\
\text { disminución } \\
\text { de los sínto- } \\
\text { mas ansiosos } \\
\text { y depresivos } \\
\text { de los pacien- } \\
\text { tes con cáncer. }\end{array}$ & $\begin{array}{l}\text { No aleatori- } \\
\text { zación. Las } \\
\text { variables } \\
\text { sociodemográ- } \\
\text { ficas no se han } \\
\text { controlado. } \\
\text { Falta de una } \\
\text { medida de } \\
\text { seguimiento. }\end{array}$ \\
\hline
\end{tabular}

5 Hace referencia al "contacto con el momento presente", componente del modelo Hexaflex postulado para la ACT (Nota de los autores).

6 Programa "Take A Breath" 


\begin{tabular}{|c|c|c|c|c|c|c|}
\hline Autores & Patología & Participantes & $\begin{array}{l}\text { Grupo } \\
\text { control }\end{array}$ & $\begin{array}{l}\text { Evaluación y } \\
\text { Seguimiento }\end{array}$ & Resultados & Limitaciones \\
\hline $\begin{array}{l}\text { Feros et } \\
\text { al. }{ }^{(12)}\end{array}$ & $\begin{array}{l}\text { Cáncer } \\
\text { de mama } \\
(48,9 \%) \\
\text { Cáncer ge- } \\
\text { nitounira- } \\
\text { rio, cabeza } \\
\text { y garganta, } \\
\text { linfoma, } \\
\text { pulmón y } \\
\text { estómago }\end{array}$ & $\begin{array}{l}45 \text { pacientes } 34 \\
\text { mujeres y } 11 \\
\text { hombres (solo } \\
28 \text { recibieron } \\
\text { el tratamiento } \\
\text { completo de } 19 \\
\text { sesiones). } \\
\text { Media de edad: } \\
5,8 \text { años ( } 25- \\
77 \text { años) }\end{array}$ & $\begin{array}{l}\text { Sin } \\
\text { grupo } \\
\text { control }\end{array}$ & $\begin{array}{l}\text { Pre-tra- } \\
\text { tamiento } \\
\text { intervención, } \\
\text { durante el } \\
\text { tratamiento, } \\
\text { post-trata- } \\
\text { miento, y } 3 \\
\text { meses des- } \\
\text { pués. }\end{array}$ & $\begin{array}{l}\text { La ACT con- } \\
\text { sigue mejoras } \\
\text { significativas } \\
\text { en el distrés, } \\
\text { estado de } \\
\text { ánimo y en } \\
\text { calidad de } \\
\text { vida, que se } \\
\text { mantienen } \\
\text { después del } \\
\text { tratamiento y } \\
\text { a los } 3 \text { meses } \\
\text { posteriores. } \\
\text { Mejora de la } \\
\text { flexibilidad } \\
\text { psicológica. }\end{array}$ & $\begin{array}{l}\text { Tamaño de } \\
\text { la muestra } \\
\text { pequeño. } \\
\text { Ausencia de } \\
\text { grupo control. } \\
\text { Posible sesgo } \\
\text { debido a la } \\
\text { deseabilidad } \\
\text { social cuando } \\
\text { se utilizan } \\
\text { medidas de } \\
\text { autoinforme } \\
\text { (evaluación } \\
\text { del estado de } \\
\text { ánimo, la cali- } \\
\text { dad de vida y } \\
\text { la flexibilidad } \\
\text { psicológica). }\end{array}$ \\
\hline
\end{tabular}

\begin{tabular}{lll}
\hline $\begin{array}{l}\text { Rost et } \\
\text { al. }{ }^{(22)}\end{array}$ & $\begin{array}{l}\text { Cáncer de } \\
\text { Ovarios }\end{array}$ & 57 mujeres \\
& & $\mathrm{M}=56$ años de \\
& & media $(32-74$ \\
& & años $)$
\end{tabular}

\begin{tabular}{|c|c|c|c|c|}
\hline $\begin{array}{l}\text { holam- } \\
\text { sseini et } \\
\text { (26) }\end{array}$ & $\begin{array}{l}\text { Cán } \\
\text { man }\end{array}$ & $\begin{array}{l}30 \text { pacientes } \\
\text { mujeres }\end{array}$ & $\begin{array}{l}\text { Sin in- } \\
\text { terven- } \\
\text { ción }\end{array}$ & $\begin{array}{l}\text { Pre y } \\
\text { post-tratam- } \\
\text { iento. }\end{array}$ \\
\hline
\end{tabular}

TAU $^{4}$ Pre-tratamien- La ACT redu- La intervento, y durante jo el estado de ción es realiel tratamiento distrés y mejo- zada en varios $\left(4^{\mathrm{a}}, 8^{\mathrm{a}}\right.$ y $12^{\mathrm{a}} \quad$ ró en la cali- lugares del sesión) dad de vida hospital. al final del tratamiento.

La ACT obtu- No todos los vo resultados participantes positivos en la completaron reducción de el tratamiento la sintomato- al que fueron logía ansiosa asignados. durante y Tamaño de la después del muestra redutratamiento cido.

\begin{tabular}{|c|c|c|c|c|c|c|}
\hline $\begin{array}{l}\text { Monte- } \\
\text { sinos et } \\
\text { al. }{ }^{(29)}\end{array}$ & $\begin{array}{l}\text { Cáncer de } \\
\text { mama }\end{array}$ & $\begin{array}{l}12 \text { mujeres } \\
M=49,4 \text { edad } \\
(31-74 \text { años }) \\
\text { DT }=14,3)\end{array}$ & $\begin{array}{l}\text { Sin in- } \\
\text { terven- } \\
\text { ción }\end{array}$ & $\begin{array}{l}\text { Pre y post- } \\
\text { tratamiento, } \\
\text { y } 1 \text { y } 3 \text { meses } \\
\text { después }\end{array}$ & $\begin{array}{l}\text { La ACT fo- } \\
\text { calizada en } \\
\text { la exposición } \\
\text { a los eventos } \\
\text { privados, re- } \\
\text { duce el miedo } \\
\text { a la recaída. } \\
\text { Los resultados } \\
\text { se mantienen } \\
\text { en el tiempo (a } \\
\text { los } 3 \text { meses) }\end{array}$ & $\begin{array}{l}\text { No se especi- } \\
\text { fican. }\end{array}$ \\
\hline
\end{tabular}


Por otro lado, los instrumentos de evaluación que se han empleado en los artículos han sido muy variados (ver Tabla 3). Para la evaluación del distrés se han empleado el Hospital Anxiety and Depression Scale (HADS), el Profile of Mood States (POMS), la Kessler Psychological Distress Scale (K10), la Depression Anxiety Stress Scale (DASS) o el Distress Thermometer.

Para evaluar las preocupaciones se han utilizaron la Parent Experience of Child Illness (PECI) y el Illness Behavior Questionnaire (IBQ).

Respecto a la ansiedad y depresión, se evaluó en todos los estudios a través del Beck Anxiety Inventory (BAI) y el Beck Depression Inventory (BDI-II), respectivamente.

En cuanto a la evaluación de la calidad de vida de los pacientes se utilizó la Functional Assessment of Cancer Therapy-General o la Functional Assessment of Cancer Therapy-Breast, dependiendo del tipo de cáncer.

En el caso de la evaluación de las habilidades cognitivas se han empleado una amplia gama de instrumentos, entre los que se encuentran el Valued Living Questionnaire (VLQ), el Acceptance amd Action Questionnaire-II (AAQ-II), el COPE, el White Bear Thought Suppression Inventory (WBSI), la Courtauld Emotional Control Scale (CECS), el Parental Psychological Flexibility Questionnaire (PPF), la MINIMAC, el Snyder Expectancy Life Questionnaire y la Mindfulness Attention Awareness Scale (MAAS).

Tabla 3. Instrumentos de las medidas principales

\begin{tabular}{|c|c|c|c|}
\hline Variable & $\begin{array}{l}\text { Instrumento de } \\
\text { Evaluación }\end{array}$ & Descripción & Artículos \\
\hline \multirow{5}{*}{$\begin{array}{l}\text { Distrés } \\
\text { Psicológico }\end{array}$} & $\begin{array}{l}\text { Hospital Anxiety and De- } \\
\text { pression Scale (HADS) }\end{array}$ & $\begin{array}{l}\text { Escala tipo Likert de } 4 \\
\text { puntos los síntomas para } \\
\text { evaluar síntomas ansiosos } \\
\text { y depresivos en pacientes } \\
\text { hospitalarios. }\end{array}$ & $\begin{array}{l}\text { Montesinos et al. }{ }^{(29)} \\
\text { Páez et al. }{ }^{(23)}\end{array}$ \\
\hline & $\begin{array}{l}\text { The Profile of Mood } \\
\text { States (POMS) }\end{array}$ & $\begin{array}{l}\text { Subescalas que evalúan } \\
\text { los estados de ánimo: } \\
\text { ansiedad, depresión, hos- } \\
\text { tilidad, confusión, vigor } \\
\text { y fatiga. }\end{array}$ & Rost et al. ${ }^{(22)}$ \\
\hline & $\begin{array}{l}\text { Depression Anxiety } \\
\text { Stress Scale (DASS) }\end{array}$ & $\begin{array}{l}\text { Escala evalúa los sínto- } \\
\text { mas depresivos, de estrés } \\
\text { y de tensión emocional. }\end{array}$ & Feros et al. ${ }^{(12)}$ \\
\hline & $\begin{array}{l}\text { The Distress Thermom- } \\
\text { eter }\end{array}$ & $\begin{array}{l}\text { Medida de autoinforme } \\
\text { sobre el grado de estrés. }\end{array}$ & Feros et al. ${ }^{(12)}$ \\
\hline & $\begin{array}{l}\text { Parents Self-Reported } \\
\text { Severety (PTSS) }\end{array}$ & $\begin{array}{l}\text { Screening indicadores del } \\
\text { estrés. }\end{array}$ & Burke et al. ${ }^{(27)}$ \\
\hline
\end{tabular}




\begin{tabular}{|c|c|c|c|}
\hline Variable & $\begin{array}{l}\text { Instrumento de } \\
\text { Evaluación }\end{array}$ & Descripción & Artículos \\
\hline \multirow{2}{*}{$\begin{array}{l}\text { Preocupa- } \\
\text { ciones }\end{array}$} & $\begin{array}{l}\text { Parent Experience of } \\
\text { Child Illness (PECI) }\end{array}$ & $\begin{array}{l}\text { Medida del ajuste emo- } \\
\text { cional: culpa y preocupa- } \\
\text { ción, recursos emociona- } \\
\text { les, dolores no resueltos, } \\
\text { incertidumbre a largo } \\
\text { plazo. }\end{array}$ & Burke et al. ${ }^{(27)}$ \\
\hline & $\begin{array}{l}\text { Illness Behavior Ques- } \\
\text { tionnaire (IBQ) }\end{array}$ & $\begin{array}{l}\text { Cuestionario que evalúa } \\
\text { la presencia de preocupa- } \\
\text { ciones acerca del cáncer } \\
\text { y el nivel de hipocondría. }\end{array}$ & Montesinos et al. ${ }^{(29)}$ \\
\hline \multirow[t]{2}{*}{ Ansiedad } & \multirow[t]{2}{*}{$\begin{array}{l}\text { Beck Anxiety Inventory } \\
\text { (BAI) }\end{array}$} & \multirow[t]{2}{*}{$\begin{array}{l}\text { Inventario de síntomas de } \\
\text { ansiedad. }\end{array}$} & $\begin{array}{l}\text { Mohabbat-Bahar et al. }{ }^{(16)} \\
\text { Gholambosseini et al. }{ }^{(26)}\end{array}$ \\
\hline & & & Rost et al. ${ }^{(22)}$ \\
\hline Depresión & $\begin{array}{l}\text { Beck Depression Inven- } \\
\text { tory (BDI-II) }\end{array}$ & $\begin{array}{l}\text { Inventario de síntomas } \\
\text { depresivos. }\end{array}$ & $\begin{array}{l}\text { Mohabbat-Bahar et al. }{ }^{(16)} \\
\text { Rost et al. }{ }^{(22)}\end{array}$ \\
\hline \multirow[b]{2}{*}{$\begin{array}{l}\text { Calidad de } \\
\text { Vida }\end{array}$} & $\begin{array}{l}\text { The Functional Assess- } \\
\text { ment of Cancer Therapy } \\
\text { - General }\end{array}$ & \multirow{2}{*}{$\begin{array}{l}\text { Evaluación de la calidad } \\
\text { de vida de los pacientes } \\
\text { con cáncer } \\
\text { Evaluación la calidad de } \\
\text { vida de los pacientes con } \\
\text { cáncer de mama. }\end{array}$} & $\begin{array}{l}\text { Feros et al. }{ }^{(12)} \\
\text { Rost et al. }{ }^{(22)}\end{array}$ \\
\hline & $\begin{array}{l}\text { The Functional Assess- } \\
\text { ment of Cancer Ther- } \\
\text { apy-Breast }\end{array}$ & & Páez et al. ${ }^{(23)}$ \\
\hline
\end{tabular}

Evaluación de las estrategias de afrontamiento

The COPE y de flexibilidad psicológica

Rost et al. ${ }^{(22)}$

Inventario sobre los pen-

Habilidades The White Bear Thought samientos que se intentan

$\begin{array}{llll}\text { Cognitivas y } & \text { Suppression Inventory } & \text { suprimir } & \text { Rost et al. }{ }^{(22)} \\ \text { Psicológicas } & \text { (WBSI) } & & \end{array}$

Escala del control emo-

cional que tienen los

$\begin{array}{ll}\text { The Courtauld Emotional } & \text { pacientes con enfermeda- } \\ \text { Control Scale (CECS) } & \text { des físicas. }\end{array}$ 


\begin{tabular}{|c|c|c|c|}
\hline Variable & $\begin{array}{c}\text { Instrumento de } \\
\text { Evaluación }\end{array}$ & Descripción & Artículos \\
\hline \multirow{9}{*}{$\begin{array}{l}\text { Habilidades } \\
\text { Cognitivas y } \\
\text { Psicológicas }\end{array}$} & $\begin{array}{l}\text { The Valued Living Ques- } \\
\text { tionnaire (VLQ) }\end{array}$ & $\begin{array}{l}\text { Cuestionario acerca del } \\
\text { número de áreas valiosas } \\
\text { afectadas. }\end{array}$ & Páez et al. ${ }^{(23)}$ \\
\hline & $\begin{array}{l}\text { Parental Psychological } \\
\text { Flexibility Questionnaire } \\
\text { (PPF) }\end{array}$ & $\begin{array}{l}\text { Cuestionario que evalúa } \\
\text { la flexibilidad psicológi- } \\
\text { ca, el bienestar emocio- } \\
\text { nal, la defusión cognitiva } \\
\text { y la aceptación en rela- } \\
\text { ción con los padres. }\end{array}$ & Burke et al. ${ }^{(27)}$ \\
\hline & $\begin{array}{l}\text { Acceptance and Action } \\
\text { Questionnaire-II (AAQ- } \\
\text { II) }\end{array}$ & $\begin{array}{l}\text { Cuestionario que mide la } \\
\text { flexibilidad psicológica } \\
\text { (la aceptación y la evita- } \\
\text { ción experiencial). }\end{array}$ & Feros et al. ${ }^{(12)}$ \\
\hline & $\begin{array}{l}\text { Snyder Expectancy Life } \\
\text { Questionnaire }\end{array}$ & $\begin{array}{l}\text { Cuestionario sobre las } \\
\text { expectativas vitales. }\end{array}$ & Ghasemi et al. ${ }^{(28)}$ \\
\hline & The COPE & $\begin{array}{l}\text { Evaluación de las estra- } \\
\text { tegias de afrontamiento } \\
\text { y de flexibilidad psico- } \\
\text { lógica }\end{array}$ & Rost et al. ${ }^{(22)}$ \\
\hline & $\begin{array}{l}\text { The White Bear Thought } \\
\text { Suppression Inventory } \\
\text { (WBSI) }\end{array}$ & $\begin{array}{l}\text { Inventario sobre los pen- } \\
\text { samientos que se intentan } \\
\text { suprimir }\end{array}$ & Rost et al. ${ }^{(22)}$ \\
\hline & $\begin{array}{l}\text { The Courtauld Emotional } \\
\text { Control Scale (CECS) }\end{array}$ & $\begin{array}{l}\text { Escala del control emo- } \\
\text { cional que tienen los } \\
\text { pacientes con enfermeda- } \\
\text { des físicas. }\end{array}$ & Rost et al. ${ }^{(22)}$ \\
\hline & $\begin{array}{l}\text { The Valued Living Ques- } \\
\text { tionnaire (VLQ) }\end{array}$ & $\begin{array}{l}\text { Cuestionario acerca del } \\
\text { número de áreas valiosas } \\
\text { afectadas. }\end{array}$ & Páez et al. ${ }^{(23)}$ \\
\hline & $\begin{array}{l}\text { Parental Psychological } \\
\text { Flexibility Questionnaire } \\
\text { (PPF) }\end{array}$ & $\begin{array}{l}\text { Cuestionario que evalúa } \\
\text { la flexibilidad psicológi- } \\
\text { ca, el bienestar emocio- } \\
\text { nal, la defusión cognitiva } \\
\text { y la aceptación en rela- } \\
\text { ción con los padres. }\end{array}$ & Burke et al. ${ }^{(27)}$ \\
\hline
\end{tabular}




\begin{tabular}{clll}
\hline Variable & \multicolumn{1}{c}{$\begin{array}{c}\text { Instrumento de } \\
\text { Evaluación }\end{array}$} & \multicolumn{1}{c}{ Descripción } & \multicolumn{1}{c}{ Artículos } \\
\hline & \multicolumn{1}{c}{$\begin{array}{l}\text { Acceptance and Action } \\
\text { Questionnaire-II (AAQ- } \\
\text { II) }\end{array}$} & $\begin{array}{l}\text { Cuestionario que mide la } \\
\text { flexibilidad psicológica } \\
\text { (la aceptación y la evita- } \\
\text { ción experiencial). }\end{array}$ & Feros et al. ${ }^{(12)}$ \\
\cline { 2 - 4 } & & & \\
$\begin{array}{c}\text { Habilidades } \\
\text { Cognitivas y } \\
\text { Psicológicas } \\
\text { (Cont.) }\end{array}$ & $\begin{array}{l}\text { Snyder Expectancy Life } \\
\text { Questionnaire }\end{array}$ & $\begin{array}{l}\text { Cuestionario sobre las } \\
\text { expectativas vitales. }\end{array}$ & Ghasemi et al. ${ }^{(28)}$ \\
& Mindfulness Attention & $\begin{array}{l}\text { Escala indicadora de la } \\
\text { capacidad de atención } \\
\text { plena. }\end{array}$ & Burke et al. ${ }^{(27)}$ \\
& & & \\
\hline
\end{tabular}

\subsection{Resultados del grupo experimental}

El valor de la variable ansiedad obtenido en los estudios de Gholambhosseini et al. ${ }^{(26)}$ $(d=6,5)$, Rost et al. ${ }^{(22)}(d=4,90)$, y Mohabbat-Bahar et al. ${ }^{(16)}(d=0,77)$, sugiere que la intervención con ACT produce una importante disminución. Sin embargo, estos resultados contrastan con lo mostrado por el grupo experimental del estudio de Páez et al. ${ }^{(23)}$, en el que se produce un cambio de tamaño medio $(d=0,55)$.

Los resultados en la variable depresión muestran un cambio de gran tamaño $(d=$ $4,36)$ tras la intervención con ACT en el estudio de Rost et al. ${ }^{(22)}$; que, no obstante, contrasta con el tamaño discreto de los estudios de Mohabbat-Bahar et al. ${ }^{(16)}(d=$ $0,65)$, y de Páez et al. ${ }^{(23)}(d=0,63)$.

En cuanto a la variable distrés, los estudios de Feros et al. ${ }^{(12)}$, y de Rost et al..$^{(22)}$, revelan una reducción elevada tras la intervención con ACT ( $d=1,00$ y de $d=3,26$, respectivamente). Sin embargo, no se muestra un cambio significativo en el estudio de Montesinos et al. ${ }^{(29)}$, que presenta un tamaño pequeño $(d=0,18)$.

En la variable calidad de vida se observa que en todos los grupos experimentales de los artículos seleccionados se produce un cambio tras la intervención. De este modo, Feros et al. ${ }^{(12)}(d=-0,51)$ muestran un aumento moderado con la intervención; mientras que Rost et al. ${ }^{(22)}(d=-3,28)$, y Páez et al. ${ }^{(23)}(d=-1,98)$, obtienen unos resultados sugerentes de una mejoría notable.

Los resultados en la variable flexibilidad psicológica son consistentes en todos los artículos seleccionados, en los que se aprecia un aumento tras la intervención con ACT, que oscila entre grande, como en Rost et al. ${ }^{(22)}(d=-4,85)$; y medio, como en los trabajos de Burke et al. ${ }^{(27)}(d=-0,67)$, y de Feros et al. ${ }^{(13)}(d=-0,63)$.

Por último, el valor de la variable expectativas ante la vida indica que después de pasar por la intervención con ACT, el grupo experimental obtiene una mejoría notable $(d=-4,00)^{(28)}$; aunque sólo se dispone de un estudio que lo avala. 
Tabla 4. Resultados en el grupo experimental (Tratamiento ACT)

\begin{tabular}{|c|c|c|c|c|c|c|c|}
\hline Variable & Artículo & $\mathbf{N}$ & Mpre & Mpost & Dtpre & Dtpost & $d$ \\
\hline \multirow{4}{*}{ Ansiedad } & Páez et al. ${ }^{(23)}$ & 38 & 11,67 & 10,17 & 2,66 & 2,93 & 0,55 \\
\hline & $\begin{array}{l}\text { Mohabbat-Ba- } \\
\text { har et al. }{ }^{(16)}\end{array}$ & 15 & 37,1 & 28,8 & 10,2 & 6,5 & 0,77 \\
\hline & $\begin{array}{l}\text { Gholam- } \\
\text { bosseini et } \\
\text { al. }^{(26)}\end{array}$ & 15 & 28,2 & 15,53 & 1,98 & 3,45 & 6,05 \\
\hline & Rost et al. ${ }^{(22)}$ & 15 & 16,88 & 5,83 & 2,13 & 1,5 & 4,90 \\
\hline \multirow{3}{*}{ Depresión } & Rost et al. ${ }^{(22)}$ & 15 & 23,14 & 8,65 & 3,14 & 2,37 & 4,36 \\
\hline & $\begin{array}{l}\text { Mohabbat-Ba- } \\
\text { har et al. }{ }^{(16)}\end{array}$ & 15 & 45,5 & 36 & 13,8 & 9,9 & 0,65 \\
\hline & Páez et al. ${ }^{(23)}$ & 38 & 10,17 & 7 & 4,96 & 3,16 & 0,63 \\
\hline \multirow{3}{*}{ Distrés } & Rost et al. ${ }^{(22)}$ & 15 & 29,91 & 2,92 & 7,82 & 5,14 & 3,26 \\
\hline & $\begin{array}{l}\text { Montesinos et } \\
\text { al. }{ }^{(29)}\end{array}$ & 8 & 18,87 & 17,25 & 8,16 & 8,37 & 0,18 \\
\hline & Feros et al. ${ }^{(12)}$ & 45 & 7,53 & 4,55 & 2,92 & 2,42 & 1,00 \\
\hline \multirow{3}{*}{$\begin{array}{c}\text { Calidad de } \\
\text { Vida }\end{array}$} & Feros et al..$^{(12)}$ & 45 & 62,68 & 72,23 & 18,33 & 15,44 & $-0,51$ \\
\hline & Páez et al. ${ }^{(23)}$ & 38 & 60 & 82,33 & 11,03 & 15,02 & $-1,98$ \\
\hline & Rost et al. ${ }^{(22)}$ & 15 & 75,63 & 89,98 & 4,14 & 3,9 & $-3,28$ \\
\hline
\end{tabular}




\begin{tabular}{|c|c|c|c|c|c|c|c|}
\hline Variable & Artículo & $\mathbf{N}$ & Mpre & Mpost & Dtpre & Dtpost & $d$ \\
\hline \multirow{3}{*}{$\begin{array}{l}\text { Flexibili- } \\
\text { dad Psico- } \\
\text { lógica }\end{array}$} & Feros et al..$^{(12)}$ & 45 & 38,38 & 45,24 & 10,66 & 10,65 & $-0,63$ \\
\hline & Burke et al. ${ }^{(27)}$ & 8 & 5,33 & 5,84 & 0,68 & 0,66 & $-0,67$ \\
\hline & Rost et al. ${ }^{(22)}$ & 15 & 12,4 & 16,04 & 0,71 & 0,48 & $-4,85$ \\
\hline $\begin{array}{l}\text { Expectativa } \\
\text { ante la vida }\end{array}$ & $\begin{array}{l}\text { Ghasemi et } \\
\text { al. }{ }^{(28)}\end{array}$ & 24 & 22,75 & 35,61 & 3,11 & 13,92 & $-4,00$ \\
\hline
\end{tabular}

Figura 2. Cambio tras la intervención en el grupo experimental

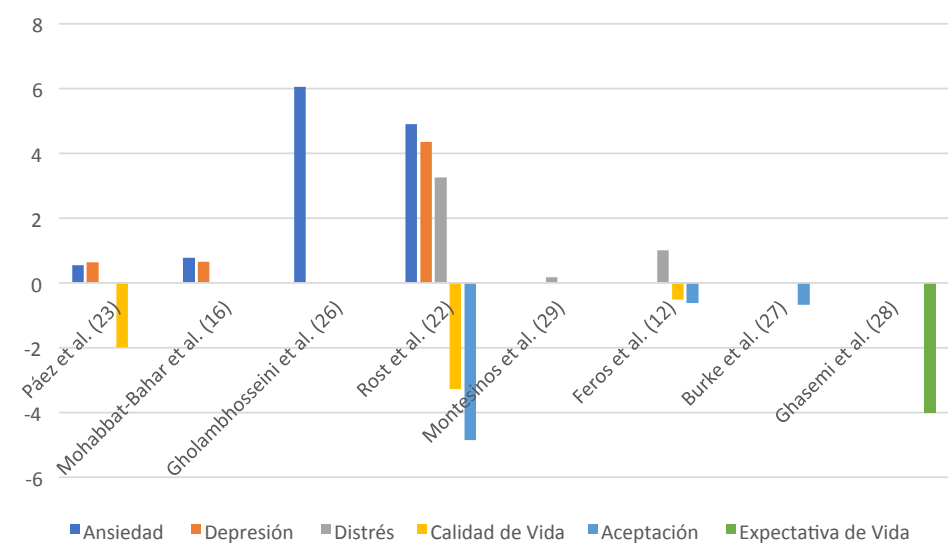

\subsection{Resultados comparativos entre el grupo experimental y el grupo control}

Al comparar los resultados entre los grupos experimentales y controles de los artículos revisados, se observa predominantemente que la intervención con ACT produce una mayor reducción en la variable ansiedad que la ausencia de tratamiento o que la intervención con terapia cognitivo-conductual. Por el contrario, en el estudio de Páez et al. ${ }^{(23)}$ la mayor reducción ocurre en el grupo control (terapia cognitivoconductual) $\left(d_{\exp }=0,55>d_{\text {control }}=0,69\right)$.

El tratamiento con ACT también se relaciona con una disminución más marcada en el nivel de la variable depresión, en contraste con los controles, en los trabajos de Rost et al. ${ }^{(22)}\left(d_{\exp }=4,36>d_{\text {control }}=2,42\right)$ y de Mohabbat-Bahar et al. ${ }^{(16)}\left(d_{\text {exp }}=0,65>d_{\text {control }}\right.$ $=-0,41)$. Sin embargo, en Páez et al. ${ }^{(23)}\left(d_{\exp }=0,63<d_{\text {control }}=1,06\right)$ el grupo control (terapia cognitivo-conductual) vuelve a mostrar una disminución más importante. 
En la variable distrés, la aplicación de la ACT disminuye el malestar emocional en mayor medida que en el grupo control, tal y como muestra el estudio de Rost et al. ${ }^{(22)}\left(d_{\exp }=3,26>d_{\text {control }}=0,71\right)$. Por contra, en el trabajo de Montesinos et al. ${ }^{(29)}$ es el grupo control (lista de espera) el que experimenta una mayor disminución de distrés $\left(d_{\text {exp }}=0,18<d_{\text {control }}=0,71\right)$.

La variable calidad de vida también aumenta en mayor medida con el tratamiento de ACT que con el control (terapia cognitivo-conductual), como así se desprende de los trabajos de Páez et al. ${ }^{(23)}\left(d_{\exp }=-1,98>d_{\text {control }}=-1,04\right)$, y de Rost et al. ${ }^{(22)}\left(d_{\text {exp }}=\right.$ $\left.-3,28>d_{\text {control }}=-0,45\right)$.

De igual forma, el tratamiento con ACT produce una mejora muy superior en flexibilidad psicológica que la que obtienen aquellos pacientes que forman parte del grupo control (terapia cognitivo-conductual), tal y como muestran los resultados de Rost et al. ${ }^{(22)}\left(d_{\text {exp }}=-4,85>d_{\text {control }}=1,46\right)$.

Por último, el tamaño del cambio en la variable expectativa ante la vida es superior en el grupo que recibió ACT, que en el grupo control (lista de espera), de acuerdo con el estudio de Ghasemi et al. ${ }^{(28)}\left(d_{\text {exp }}=-4.00>d_{\text {control }}=-0.10\right)$.

Los resultados de las diferencias entre los grupos experimentales y controles en las variables dependientes estudiadas se representan en la Tabla 4, y en las Figuras $3,4,5,6,7$ y 8 .

Tabla 5. Resultados de la interacción entre el grupo control y el grupo experimental

\begin{tabular}{|c|c|c|c|c|}
\hline Variable Dependiente & Artículo & Tipo de Control & Dexp & Dcontrol \\
\hline \multirow{4}{*}{ Ansiedad } & Páez et al. ${ }^{(23)}$ & $\begin{array}{l}\text { Terapia Cogniti- } \\
\text { vo-Conductual }\end{array}$ & 0,55 & 0,69 \\
\hline & $\begin{array}{c}\text { Mohabbat-Bahar et } \\
\text { al. }^{(16)}\end{array}$ & Lista de Espera & 0,77 & $-0,26$ \\
\hline & $\begin{array}{c}\text { Gholambosseini et } \\
\text { al. }^{(26)}\end{array}$ & Lista de Espera & 6,05 & 2,20 \\
\hline & Rost et al. ${ }^{(22)}$ & $\begin{array}{l}\text { Protocolo de } \\
\text { intervención } \\
\text { TAU }\end{array}$ & 4,90 & 1,92 \\
\hline \multirow{3}{*}{ Depresión } & Rost et al. ${ }^{(22)}$ & $\begin{array}{l}\text { Protocolo de } \\
\text { Intervención } \\
\text { TAU }\end{array}$ & 4,36 & 2,42 \\
\hline & $\begin{array}{l}\text { Mohabbat-Bahar et } \\
\text { al. }^{(16)}\end{array}$ & Lista de Espera & 0,65 & $-0,41$ \\
\hline & Páez et al. ${ }^{(23)}$ & $\begin{array}{l}\text { Terapia Cogniti- } \\
\text { vo-Conductual }\end{array}$ & 0,63 & 1,06 \\
\hline
\end{tabular}




\begin{tabular}{|c|c|c|c|c|}
\hline Variable Dependiente & Artículo & Tipo de Control & Dexp & Dcontrol \\
\hline \multirow{2}{*}{ Distrés } & Rost et al. ${ }^{(22)}$ & $\begin{array}{l}\text { Protocolo de } \\
\text { Intervención } \\
\text { TAU }\end{array}$ & 3,26 & 0,71 \\
\hline & Montesinos et al. ${ }^{(29)}$ & Lista de Espera & 0,18 & 0,71 \\
\hline \multirow[b]{2}{*}{ Calidad de Vida } & Páez et al. ${ }^{(23)}$ & $\begin{array}{l}\text { Terapia Cogniti- } \\
\text { vo-Conductual }\end{array}$ & $-1,98$ & $-1,04$ \\
\hline & Rost et al. ${ }^{(22)}$ & $\begin{array}{l}\text { Protocolo de } \\
\text { Intervención } \\
\text { TAU }\end{array}$ & $-3,28$ & $-0,45$ \\
\hline Flexibilidad Psicológica & Rost et al. ${ }^{(22)}$ & $\begin{array}{l}\text { Protocolo de } \\
\text { Intervención } \\
\text { TAU }\end{array}$ & $-4,85$ & 1,46 \\
\hline Expectativa ante la vida & Ghasemi et al. ${ }^{(28)}$ & Lista de Espera & $-4,00$ & $-0,10$ \\
\hline
\end{tabular}

Figura 3. Resultados del grupo experimental y control en la variable ansiedad

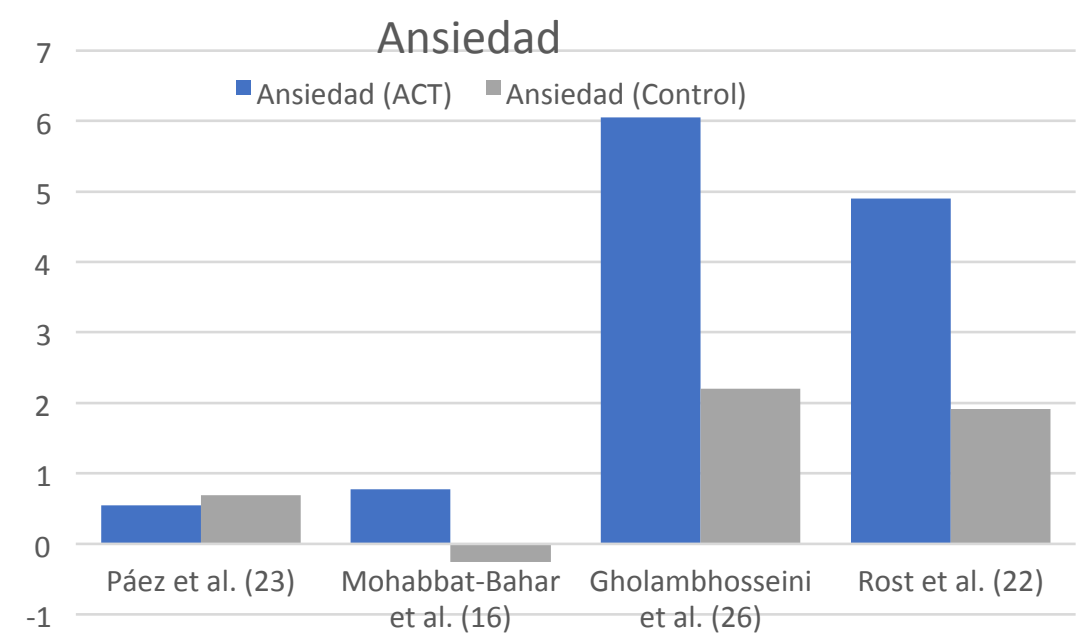


Figura 4. Resultados del grupo experimental y control en la variable depresión

\section{Depresión}

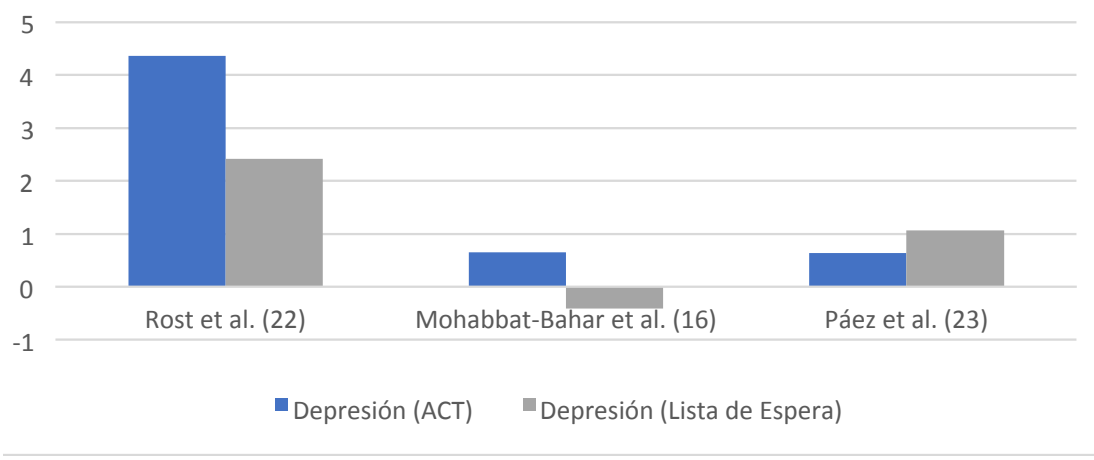

Figura 5. Resultados del grupo experimental y control en la variable distrés Distrés

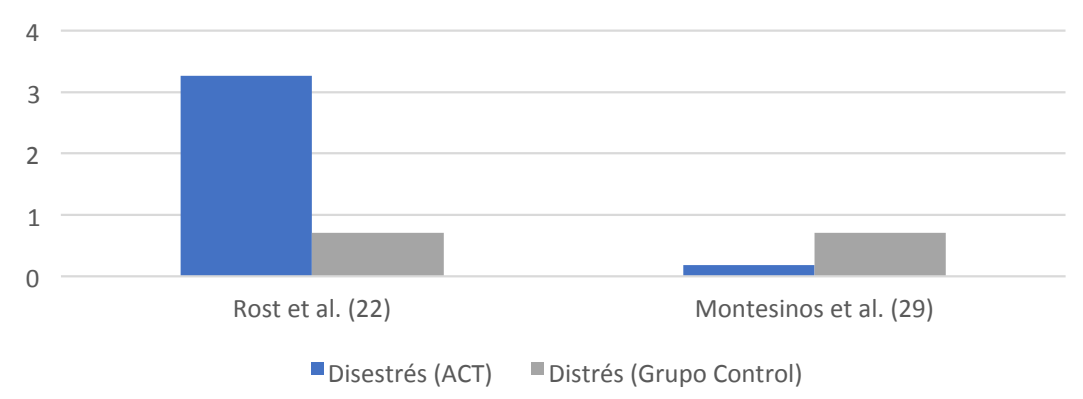

Figura 6. Resultados del grupo experimental y control en la variable calidad de vida.

\section{Calidad de Vida}

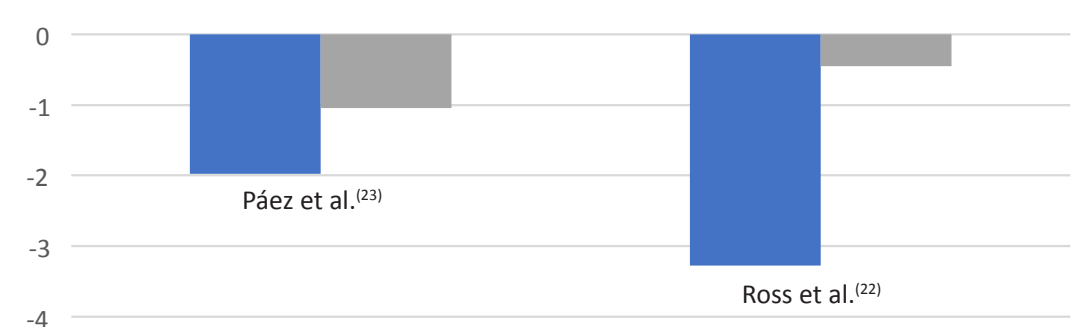

- Calidad de Vida (ACT) $\quad$ Calidad de Vida (Control) 
Figura 7. Resultados del grupo experimental y control en la variable aceptación

\section{Aceptación}

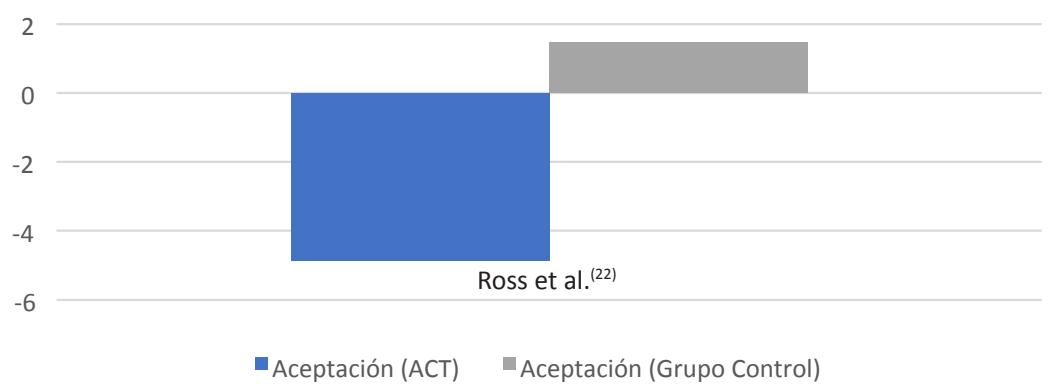

Figura 8. Resultados del grupo experimental y control en la variable expectativa de vida

\section{Expectativa de Vida}

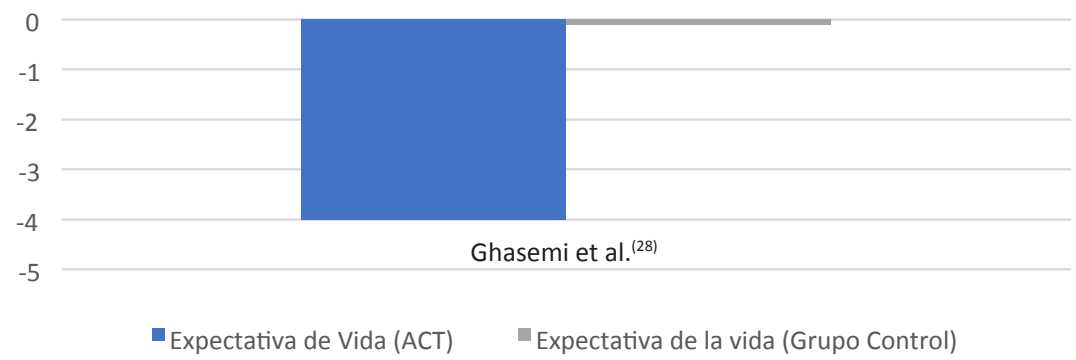

\section{Discusión}

Recibir un diagnóstico de cáncer es un evento que cambia la vida de cualquier persona. Los pacientes de cáncer pasan por incontables situaciones nuevas, que llevan consigo un alto nivel de estrés a causa de las preocupaciones, temores, dolores y síntomas depresivos asociados ${ }^{(2,12)}$. También sus familiares se ven envueltos en el curso de la enfermedad, al tener que proporcionar cuidado y apoyo emocional al enfermo, obligándoles a asumir nuevas responsabilidades ${ }^{(17)}$.

El tipo de intervención psicológica más utilizada y estudiada con pacientes de cáncer se encuadraría dentro de las terapias cognitivo-conductuales, también conocidas como terapias de "segunda generación" (7). Su objetivo principal consiste en modificar directamente el comportamiento (cogniciones, emociones y conducta observable), habiendo resultado ser especialmente eficaces cuando el paciente presenta un estilo de afrontamiento pasivo/evitativo o de negación ${ }^{(3)}$. Sin embargo, la mejoría alcanzada por los pacientes en la reducción de los niveles sintomáticos tras finalizar la intervención se desvanece con el paso del tiempo ${ }^{(5)}$.

Como alternativa a ello, en las últimas dos décadas están emergiendo con fuerza las terapias de "tercera generación", cuyo principal exponente sería la ACT, y que 
conceptualiza la cognición y el lenguaje desde una perspectiva contextual y funcional, explicando de forma empírica su relación con los patrones de comportamiento observables $^{(9)}$. A diferencia de lo propuesto por las terapias de "segunda generación", la ACT busca que el paciente tome distancia de sus eventos privados aversivos (pensamientos, emociones y sensaciones molestas), aceptándolos, para que así pueda reorientar su actuación a lo que considere importante en su vida, de acuerdo con sus valores (direcciones con significado) ${ }^{(23)}$. Estas actuaciones, que tienen en común promover el distanciamiento de los fenómenos mentales (defusión cognitiva) y suprimir los intentos de control emocional y cognitivo, han mostrado una eficacia superior a las terapias cognitivo-conductuales en el tratamiento de las alteraciones psicológicas de los pacientes de cáncer, así como en el mantenimiento de la mejoría en el largo plazo ${ }^{(22,23)}$.

No obstante, las investigaciones y estudios realizados sobre la aplicación de la $\mathrm{ACT}$ en pacientes con cáncer son escasas hasta la fecha, como también han señalado Graham et al. ${ }^{(9)}$, no encontrándose ningún estudio de revisión sistemática ni de metaanálisis al respecto. Esto es debido a la relativa juventud de la ACT, cuyas publicaciones no suelen tener más de 15 años de antigüedad, lo que contribuiría a que su número fuera menor en comparación con el de otras investigaciones realizadas desde las aproximaciones terapéuticas de "primera" o "segunda generación", que llevan más años en la escena psicológica.

Se decide, por tanto, llevar a cabo una revisión sistemática sobre los estudios empíricos publicados en revistas de lengua inglesa y española, que permita establecer el estado actual de la cuestión. La búsqueda de las publicaciones que reunieran los criterios de calidad científica y relevancia al objeto de estudio, arrojaron un total de 8 artículos. En ellos, en cuanto al tipo de tumor, el $75 \%$ de los estudios versan sobre el cáncer de mama, mientras que el $25 \%$ hacen referencia al cáncer de ovarios y otros tipos de cáncer. Esta preponderancia puede deberse a la mayor prevalencia del cáncer de mama sobre otro tipo de cáncer en la población general. De hecho, es el segundo tipo de cáncer más frecuente, tras el de pulmón, según el último informe de 2017 de la Sociedad Española de Oncología Médica (SEOM) ${ }^{(30)}$.

El análisis estadístico de los resultados encontrados a través de la $d$ de Cohen demuestra que la intervención desde la $\mathrm{ACT}$ en pacientes con cáncer conlleva una mejoría entre media y alta de la sintomatología ansiosa y depresiva, el distrés emocional, la calidad de vida, la flexibilidad psicológica y las expectativas ante la vida. En particular, considerando el tamaño del efecto en cada una de estas variables, los mayores cambios se obtienen en este orden: expectativas ante la vida, ansiedad, flexibilidad psicológica, calidad de vida, depresión y distrés. Estos resultados son consistentes con lo señalado años atrás por Luciano et al. ${ }^{(7)}$.

De igual modo, comparando el tamaño del efecto mostrado por la intervención con ACT con intervenciones cognitivo-conductuales (que incluyen componentes de entrenamiento en relajación, resolución de problemas y reestructuración cognitiva) o lista de espera, se halla una superioridad de la primera, de una magnitud entre media y alta, en las variables expectativas ante la vida, flexibilidad psicológica, calidad de vida, distrés, depresión y ansiedad.

La ansiedad es una de las principales experiencias emocionales aversivas que suele estar incrementada tras el diagnóstico de cáncer, pudiendo derivar en trastornos de ansiedad por las conductas de escape y/o evitación que fomentan. Algunas investigaciones sostienen que los trastornos de ansiedad se desarrollan y 
se mantienen por el comportamiento de evitación de los síntomas ansiosos y por la fusión con el pensamiento ${ }^{(26)}$. De este modo, desde la ACT se procura que el paciente acepte esas experiencias emocionales en vez de eliminarlas, como se haría desde otro enfoque de la psicoterapia. No obstante, la aceptación no significa resignación, aguante o fastidio, sino una elección de una postura abierta, receptiva y flexible con respecto a las experiencias del momento ${ }^{(6)}$.

En consonancia con lo anterior, los resultados revisados revelan que la mejoría en la flexibilidad psicológica se relaciona con una mayor aceptación de pensamientos, sentimientos, sensaciones, recuerdos e imágenes disfóricos, que los pacientes de cáncer tienden a evitar o controlar. Es esta aceptación y disposición abierta a experimentar los eventos privados molestos, al servicio de las acciones valiosas que dan sentido a la vida de la persona ${ }^{(6)}$, la que podría explicar que los resultados se mantengan a largo plazo y que se evite la cronicidad del curso de la sintomatología, como muestran los trabajos de Feros et a. ${ }^{(12)}$, y Luciano et al. ${ }^{(7)}$, en los que la ACT ha resultado ser más eficaz a largo plazo cuando se compara con tratamientos cognitivos, condiciones placebo o lista de espera.

A pesar de todo lo explicado con anterioridad, existe una contradicción en los artículos seleccionados entre, por una parte, los fundamentos teóricos de la ACT y, por otra, su puesta en práctica para el tratamiento de los problemas psicológicos de los pacientes de cáncer. Si el objetivo principal de la ACT no es la eliminación de los síntomas, sino la mejora de la flexibilidad psicológica hacia los pensamientos y sentimientos que surjan, no siendo relevantes los métodos y técnicas empleados ${ }^{(6,7)}$; el hecho de que muchos artículos traten de disminuir los niveles de ansiedad y depresión, o traten de medir la sintomatología, entraría en contradicción con los principios en los que se basa la ACT, y les situaría más cerca del modelo médico subyacente a las terapias de segunda generación, ante las que se presenta como alternativa.

\subsection{Limitaciones}

El presente trabajo también muestra algunas limitaciones, como el número reducido de los artículos que sigan diseños experimentales o cuasiexperimentales, lo cual restringe la solidez de las conclusiones acerca de la eficacia de la ACT en el ámbito oncológico; aunque también es cierto que el análisis de los datos publicados muestra resultados prometedores y parece inequívoca la mejoría que promueven.

Otra limitación de este trabajo ha sido la heterogeneidad de los grupos controles utilizados (terapia cognitivo-conductual, lista de espera). Además, la presencia en los artículos seleccionados de una muestra reducida de enfermedades oncológicas (cáncer de mama, genitourinario, cabeza, garganta, linfoma, pulmón y estómago), puede hacer variar los resultados si se estudiaran en otro tipo de tumores, ya que los pacientes con distintos tipos de cáncer pueden experimentar vivencias y síntomas psicológicos muy diferentes entre sí, respecto a su intensidad, duración, localización, significado y discapacidad, al igual que por el pronóstico y el tratamiento recibido. De igual manera, la sobrerrepresentación de los artículos revisados de cáncer de mama (75\%) frente al resto (25\%), podría sesgar las conclusiones acerca de la eficacia de la ACT en las variables psicológicas estudiadas.

Por otro lado, el hecho de que cada uno de los estudios continúe el seguimiento con diferente temporalidad, hace que las conclusiones de muchos de ellos respecto 
al mantenimiento de las mejorías no se puedan tomar como definitivas, al no poder compararse en las mismas condiciones.

En resumen, para futuras revisiones y/o estudios de metaanálisis sería aconsejable contar con un número mucho mayor de artículos, en los que se dispusiera de muestras de pacientes más numerosas, de modo que permitan extraer conclusiones inequívocas y de mayor robustez. Así, otra manera de mejorar esta revisión sería limitar los artículos atendiendo al grupo control con el que se pueda comparar las puntuaciones tras la intervención, además de agrupar los estudios en cuanto a la temporalidad del seguimiento. Esto podría contribuir a que los resultados sean más fiables a la hora de determinar la eficacia de la ACT en pacientes de cáncer.

\section{Conclusiones}

- La mayoría de los estudios de ACT en pacientes de cáncer han versado sobre el cáncer de mama hasta el momento.

- Los pacientes adultos son la población más prevalente en las muestras de los estudios encontrados sobre la utilización de ACT en el cáncer, aunque también se han encontrado estudios que tienen en cuenta a los familiares de estos pacientes.

- La ACT ha resultado más eficaz en comparación con las distintas terapias psicológicas con las que se ha comparado (terapia cognitivo conductual, protocolo TAU), aunque debe tenerse en cuenta que el tipo de grupo de control es muy heterogéneo en los distintos artículos.

- Los resultados muestran una disminución moderada de la sintomatología de ansiedad, depresión y distrés en pacientes diagnosticados de cáncer.

- Los resultados señalan mejoras en la calidad de vida, expectativas ante la vida y aceptación, en los pacientes tras aplicar la ACT.

- Los resultados de todos los artículos muestran una mejoría a medio-largo plazo en la flexibilidad psicológica, la aceptación, la defusión del pensamiento, una mayor clarificación de los valores y compromiso de actuar según estos en los pacientes tras finalizar el tratamiento de ACT.

- La mayoría de los estudios consideran que la ACT ha resultado ser más eficaz a largo plazo en el mantenimiento de la mejoría apreciada en los pacientes, mientras que no se encuentran tantas diferencias justo al finalizar la intervención.

- A pesar de las limitaciones encontradas en este estudio, tales como el limitado número de estudios analizados, la heterogeneidad de los grupos controles y la diferencia en la temporalidad de seguimiento, se han obtenido resultados prometedores para futuras revisiones y/o metaanálisis.

- Esta revisión informa sobre la necesidad de incrementar el número de estudios que evalúen la eficacia de la ACT sobre los pacientes diagnosticados de cáncer y familiares.

\section{Referencias bibliográficas}

1. Ruiz L, Callejón Z. Terapias conductuales de tercera generación en personas mayores: una revisión de la literatura. Psychol Soc Educ 2014;6:113-24. 
2. Luciano C, Páez M. Terapia de aceptación y compromiso (ACT) aplicada a pacientes con cáncer. En: Cruzado J, editor. Manual de psicooncología. Tratamientos en pacientes con cáncer. Madrid: Pirámide; 2013. p. 261-80.

3. Font A, Rodríguez E. Eficacia de las intervenciones psicológicas en mujeres con cáncer de mama. Psicooncologia 2007;4:423-46.

4. Pérez M. La psicoterapia desde el punto de vista conductista. Madrid: Biblioteca Nueva; 2013.

5. Páez M, Luciano C, Gutiérrez O. La aplicación de la Terapia de Aceptación y Compromiso (ACT) en el tratamiento de problemas psicológicos asociados al cáncer. Psicooncologia 2005;2:49-70.

6. Pérez M. Las terapias de tercera generación como terapias contextuales. Madrid: Síntesis; 2014.

7. Luciano C, Valdivia M. La Terapia de Aceptación y Compromiso (ACT). Fundamentos, características y evidencia. Pap Psicol 2006;27:79-91.

8. Ruiz F. A review of Acceptance and Commitment Therapy (ACT) empirical evidence: correlational, experimental psychopathology, component and outcome studies. Rev Int Psicol Ter Psicol 2010;10:125-62.

9. Graham CD, Gouick J, Krahé C, Guillanders D. A systematic review of the use of Acceptance and Commitment Therapy (ACT) in chronic disease and long-term conditions. Clin Psychol Rev 2016;46:46-58.doi:10.1016/j.cpr.2016.04.009

10. Barraca J. La Terapia de Aceptación y compromiso (ACT). Fundamentos, aplicación en el contexto clínico y áreas de desarrollo. Misc Comillas 2007;65:761-81.

11. Wilson K, Luciano C. Terapia de Aceptación y Compromiso (ACT). Un tratamiento conductual orientado a los valores. Madrid: Pirámide; 2002.

12. Feros D, Lane L, Ciarrochi J, Blackledge J. Acceptance and Commitment Therapy (ACT) for improving the lives of cancer patients: a preliminary study. Psychooncology 2013;22:459-64. doi:10.1002/pen.2083

13. Mitchell A, Chan M, Bhatti H. Prevalence of depression, anxiety, and adjustment disorder in oncological, hematological, and palliative-care settings: a meta-analysis of 94 interview - based studies. Lancet Oncol 2011;12:160-74. doi: 10.1016/S1470-2045(11)70002-X

14. Osborn R, Demoncada A. Psychosocial interventions for depression, anxiety, and quality of life in cancer survivors: meta-analyses. Psychiatry Med 2006;36:13-34.

15. Hyunsung Oh M, Kathleen Eli D, Subica A. Depression and family interaction among low-income, predominantly hispanic cancer patients: a longitudinal analysis. Support Care Cancer 2014;22:427-34. doi:10.1007/s00520-013-1993-2

16. Mohabbat-Bahar S, Maleki-Rizi F, Akbari M, Moradi-Joo M. Effectiveness of group training based on Acceptance and Commitment Therapy on anxiety and depression of women with breast cancer. Iran J Cancer Prev 2015;8:71-6.

17. Köhle N, Drossaert C, Schreurs K, Hagedoorn M, Verdonck-de Leeuw I, Bohlmeijer E. A web - based self-help intervention for partners of cancer patients based on Acceptance and Commitment Therapy: a protocol of a randomized controlled trial. BMC Public Health 2015;15:303. doi:10.1186/s12889-015-1656-y

18. Patterson P, McDonald F, Ciarrochi J, Hayes L, Tracey D, Wakefield C, White K. A study protocol for Truce: a pragmatic controlled trial of a seven - week acceptance and commitment therapy program for young people who have parent with cancer. BMC Psychol 2015;3:31. doi:10.1186/s40359-015-0087-y 
19. Visser A, Huizinga G, Van der Graaf W, Hoekstra H, Hoeskstra-Weebers J. The impact of parental cancer on children and the family: a review of the literature. Cancer Treat Rev 2004;30:683-94. 10.1016/j.ctrv.2004.06.001

20. Van den Hurk D, Schellekens M, Molema J, Speckens A, Van der Drift M. MindfulnessBased Stress Reduction for lung cancer patients and their partners: results of a mixed methods pilot study. Palliat Med 2015;29:652-60. doi:10.1177/0269216315572720

21. Moreira H, Canavarro M. Psychosocial adjustment and marital intimacy among partners of patients with breast cancer: a comparison study with partners of healthy women. J Psychosoc Oncol 2015;31:282-304. doi:10.1080/07347332.2013.778934

22. Rost A, Wilson K, Buchanan E, Hildebrandt M, Mutch D. Improving psychological adjustment among late-state ovarian cancer patients: examining the role of avoidance in treatment. Cogn Behav Pract 2012;19:508-17. 10.1016/j.cbpra.2012.01.003

23. Páez M, Luciano C, Gutiérrez O. Tratamiento psicológico para el afrontamiento del cáncer de mama. Estudio Comparativo entre estrategias de aceptación y de control cognitivo. Psicooncologia 2007;4:75-95.

24. Pardo A. Análisis de Datos en ciencias sociales y de la salud I. Madrid: Síntesis; 2014.

25. Cohen J. A Power Primer. Psychol Bull 1992;112:155-9.

26. Gholamhosseini S, Mojtabaie M. Effectiveness of Acceptance and Commitment Therapy (ACT) to reduce the symptoms of anxiety in women with breast cancer. J Int Rev Life Sci 2014;4:27-31.

27. Burke K, Muscara F, McCarthy M, Dimovski A, Hearps S, Anderson V, Walser R. Adapting Acceptance and Commitment Therapy for parents of children with life threatening illness: pilot study. Fam Syst 2014;32:122-7. doi: 10.1037/fsh0000012.

28. Ghasemi F, Dehghan F, Farnia V, Tatari F, Alikhani M. Effectiveness of Acceptance and Commitment Therapy on life expectancy of female cancer patients at Thran's Dehshpour Institute in 2015. Asian Pac J Cancer Prev 2016;17:4113-6. doi: 0.14456/apjcp.2016.223/ APJCP.2016.17.8.4113

29. Montesinos F, Luciano C. Acceptance of relapse fears in breast cancer patients: effects of an ACT-based abridged intervention. Psicooncologia 2016;13:7-21. doi:10.5209/rev_ PSIC.2016.v13.n1.52484

30. Sociedad Española de Oncología Médica. Las cifras del cáncer en España [Internet]; 2017. [Acceso 1 de junio de 2018] Disponible en: https://www.seom.org/es/prensa/elcancer-en-espanyacom/105941-las-cifras-del-cancer-en-espana-2017?showall=1 
